\title{
Delayed Cavernous Carotid Artery Pseudoaneurysm Caused by Absorbable Plate Following Transsphenoidal Surgery: Case Report and Review of the Literature
}

\author{
Alexander Tuchman ${ }^{1} \quad$ Alexander A. Khalessi ${ }^{1} \quad$ Frank J. Attenello ${ }^{1}$ Arun P. Amar ${ }^{1}$ Gabriel Zada ${ }^{1}$ \\ ${ }^{1}$ Department of Neurosurgery, University of Southern California, \\ Los Angeles, California, USA \\ J Neurol Surg Rep 2013;74:10-16. \\ Address for correspondence Alexander Tuchman, Department of \\ Neurosurgery, University of Southern California, 1200 N. State Street, \\ Suite 3300, Los Angeles, CA 90033, USA \\ (e-mail: alexandertuchman@gmail.com).
}

\begin{abstract}
Keywords

- cavernous

- carotid

- pseudoaneurysm

- artery

\section{Introduction}

The transsphenoidal approach is the most commonly utilized operation for the surgical treatment of sellar lesions and is a relatively safe operation in experienced centers. ${ }^{1}$ Following resection of pituitary adenomas and other sellar tumors, many surgeons utilize absorbable plates to reconstruct the bony sellar floor to serve as a buttress for the sellar contents and repair construct. Although usually safe, vascular injury in conjunction with insertion of rigid plates following sellar tumor resection has been described once before. ${ }^{2}$
\end{abstract}

Background Bioabsorbable plates are frequently utilized in the repair of skull base defects following transsphenoidal operations. Traumatic intracranial pseudoaneurysms are a rare complication of transsphenoidal surgery. To date, iatrogenic carotid pseudoaneurysm associated with the use of an absorbable plate has been reported once. Results A 57-year-old man with a large nonfunctional pituitary macroadenoma underwent an endoscopic transsphenoidal operation with gross total resection. An absorbable plate was placed extradurally to reconstruct the sellar floor. He experienced delayed repeated epistaxis, followed by a right middle cerebral artery distribution embolic stroke. Computed tomorgraphy (CT) angiogram 6 weeks postoperatively revealed a $6 \times 4 \mathrm{~mm}$ pseudoaneurysm located on the medial wall of the right cavernous internal carotid artery. Stent coiling was used to successfully obliterate the pseudoaneurysm, and the patient fully recovered.

Conclusion Delayed erosion of the carotid artery wall caused by a plate used to reconstruct the sellar floor may manifest with epistaxis or embolic stroke. The authors' preference is to avoid insertion of a rigid plate for sellar floor reconstruction in the absence of intraoperative cerebrospinal fluid (CSF) leaks, unless it is required to buttress a large skull base defect. Short-segment embolization with stent coiling is the preferred treatment option for carotid pseudoaneurysms following transsphenoidal operations.

Common complications of transsphenoidal operations include endocrine abnormalities and cerebrospinal fluid (CSF) leaks. ${ }^{3}$ Vascular injury is a rare but serious complication of transsphenoidal surgery encountered in 0.8 to $1.1 \%$ of cases, with an associated mortality of nearly $30 \%{ }^{4-6}$ The majority of vascular injuries are identified at the time of surgery, usually resulting from direct injury to the internal carotid artery during resection of tumor within the cavernous sinus or upon opening of the dura, often resulting in profuse arterial hemorrhage. ${ }^{6-9}$ Other described vascular complications include vasospasm, carotid thrombosis,

(c) 2013 Georg Thieme Verlag KG Stuttgart · New York
DOI http://dx.doi.org/ 10.1055/s-0033-1338164. ISSN 2193-6358. 


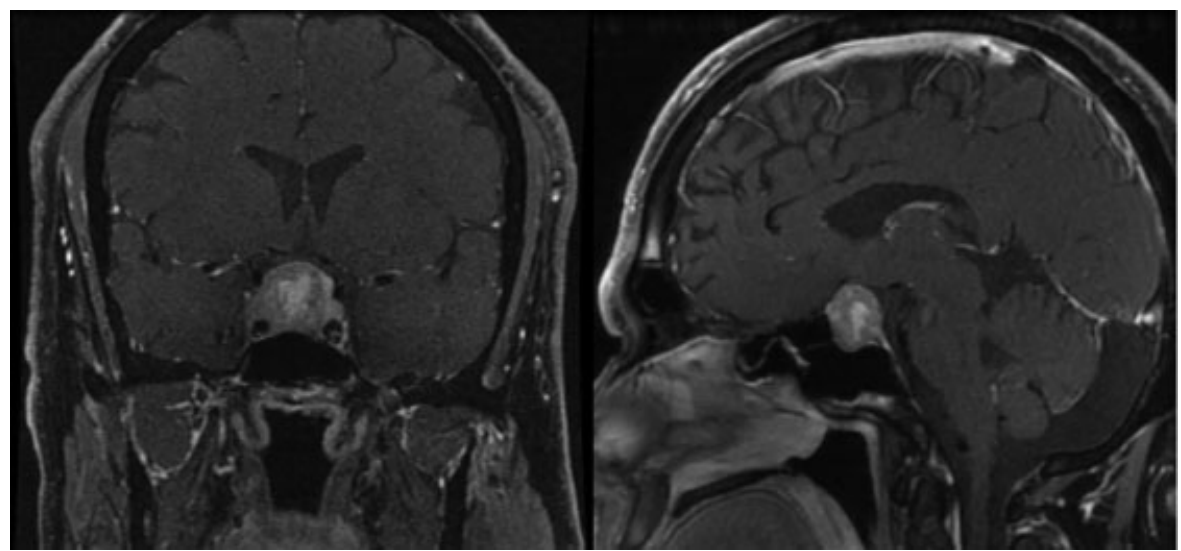

Fig. 1 Preoperative MRI Sella with contrast.

cavernous sinus thrombosis, embolism, caroticocavernous fistula, or pseudoaneurysm. ${ }^{2,3,7,8,10-19}$

Postoperative carotid pseudoaneurysm, though rare, represents a grave risk to the patient if unrecognized. It may lead to delayed hemorrhagic or embolic complications when the patient is no longer in a monitored hospital setting. This case report highlights the importance of rapid diagnosis and treatment of these lesions. We present a rare case of delayed pseudoaneurysm and embolic stroke following erosion of a rigid plate into the cavernous internal carotid artery.

\section{Case Report}

A 57-year-old man with a nonfunctional pituitary macroadenoma causing vision loss underwent a gross total, endoscopic transsphenoidal resection (-Fig. 1). The tumor was invading the right cavernous sinus wall. During the procedure to resect the tumor from this region, there was some venous bleeding that was easily controlled by temporarily packing the area using Gelfoam (Pfizer, New York, New York, USA) with thrombin. Following resection, a custom-fit bioabsorbable plate was placed extradurally to reconstruct the sellar floor. The patient was discharged home on postoperative day 2 in excellent condition. Four weeks later, he experienced epistaxis for which he was treated at an outside emergency department. The bleeding was controlled with nasal packs, and the patient was discharged home. Six weeks following the operation, he presented to clinic with dysarthria as well as left hand and facial weakness and was admitted for further work-up. Neuroimaging revealed subacute infarcts in right middle cerebral artery distribution. Computed tomography (CT) angiogram showed a $6 \times 4 \mathrm{~mm}$ pseudoaneurysm located on the medial wall of the right cavernous internal carotid artery. A hypodensity likely representing the implanted absorbable plate was noted to be compressing the right carotid artery in this region (-Fig. 2). Stent coiling with placement of eight detachable coils was used to successfully obliterate the pseudoaneurysm (-Fig. 3). Follow-up digital subtraction angiogram and CT angiogram demonstrated durable obliteration of the pseudoaneurysm with preserved flow through the carotid artery. On follow-up, the patient's neurological deficits have resolved.

\section{Discussion}

Iatrogenic carotid pseudoaneurysm is an extremely rare complication of transsphenoidal surgery, with only 24 reported cases found in our review of the literature. Only one previous case was associated with sellar floor reconstruction. ${ }^{2}$ A pseudoaneurysm is caused by injury to the carotid artery wall leading to an encapsulated hematoma in communication with the ruptured artery. In general, an aneurysmal rupture of the cavernous carotid will result in a caroticocavernous fistula. However, severe epistaxis and pituitary apoplexy have been reported in patients whose medial cavernous sinus wall has been eroded by tumor or violated during surgery. ${ }^{4}$ Thus, the consequences of aneurysmal rupture support urgent treatment of even incidentally diagnosed lesions, as they represent a grave risk to the postoperative

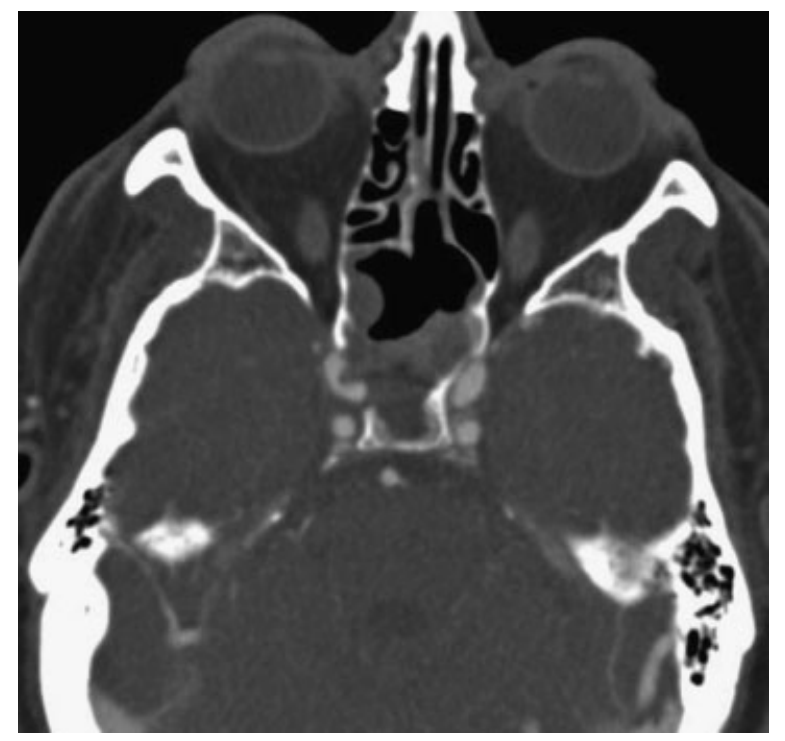

Fig. 2 CT-angiogram of the brain showing hypodense absorbable plate compressing the right cavernous carotid artery. 


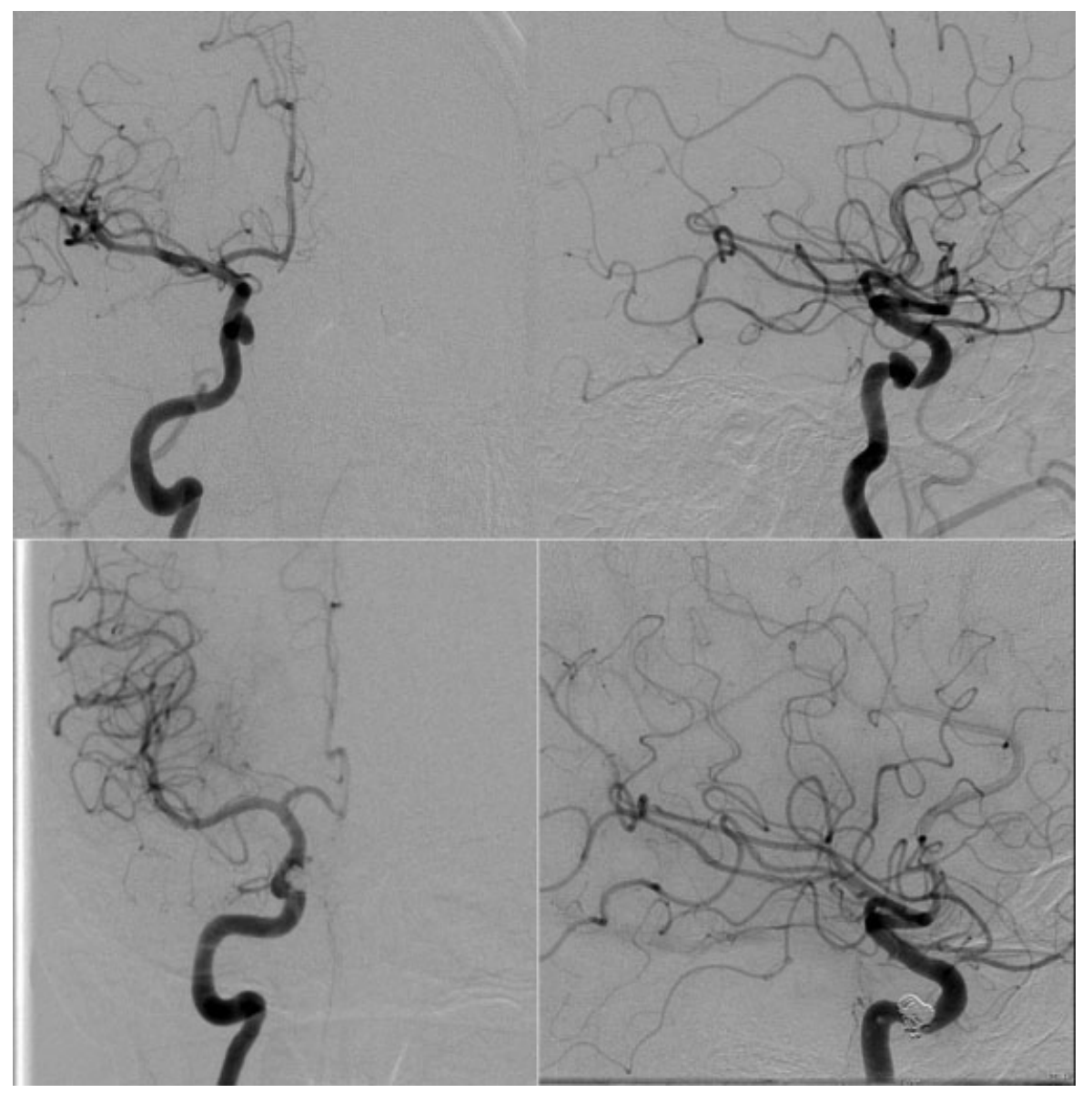

Fig. 3 Digital subtraction angiogram showing right cavernous carotid pseudoaneurysm before and after stent-assisted coil embolization.

patient. Because of the extremely low reported incidence of pseudoaneurysms after transsphenoidal surgery, postoperative angiography is not routinely performed. ${ }^{6}$ Although in cases where there is significant arterial bleeding or concern for carotid injury, many experts recommend immediate postoperative angiography. 6,15

Based on the uneventful operative course and features observed on CT angiogram, the pseudoaneurysm was likely caused by erosion of the absorbable plate into the cavernous carotid artery. This has been previously reported by Crowley et al and was also associated with delayed severe epistaxis followed by transient ischemic deficits. ${ }^{2}$ Despite its low complication rates, these cases highlight the risks associated with sellar floor reconstruction. ${ }^{20}$ Both patients were treated via endovascular means with good results. In a majority of cases, the absorbable plates used to reconstruct the bony sellar floor can be custom fit to approximate the size of the defect. The surgeon must take extra care to ensure that the cut edges are as round and smooth as possible, and that the size of the plate is just large enough to be inserted beneath the bony edges of the lateral sellar floor, and in an extradural fashion. Perhaps more importantly, the authors now make every attempt to only use a rigid buttress when necessary-that is, only in cases where intraoperative CSF leaks are observed in the setting of a large bony defect that cannot be repaired or adequately buttressed with routine techniques, including cellulose sponge, Duragen (Integra LifeSciences Corporation, Plainsboro, New Jersey, USA), and fibrin glue.

According to our search of the literature, 18 publications reporting 24 cases of iatrogenic carotid pseudoaneurysm following transsphenoidal surgery were identified (-Table 1). Among published cases, the mean patient age was 45.6 (range 22 to 74 ), with a slight female predominance (13/22 patients). Intraoperative arterial hemorrhage requiring packing was described in 17 of 24 cases; $21 \%$ of cases reported the operation as uneventful. We recommend additional delayed imaging in patients with high suspicion for arterial injury, considering multiple reports in which the immediate postoperative angiogram was negative, only to have the patient present with a pseudoaneurysm in a delayed fashion. ${ }^{16,17,21}$ Common presentations prior to diagnosis included severe epistaxis, ${ }^{10}$ postoperative high-suspicion angiogram, ${ }^{10}$ routine follow-up imaging, ${ }^{2}$ and cranial neuropathy. ${ }^{1}$ The interval to diagnosis following surgery varied between 0 days and 10 years. Delay to diagnosis may lead to aneurysmal rupture or embolic stroke outside of a monitored setting. In fact, in nine cases reported in the literature, the patient was discharged from the hospital prior to the diagnosis being made.

The treatment options for cavernous carotid pseudoaneurysms include open or endovascular surgery. Although conservative management may be an option, one report in which 


\begin{tabular}{|c|c|c|c|c|c|c|c|c|c|c|c|c|c|}
\hline 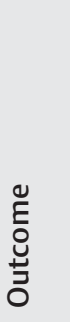 & 告 & 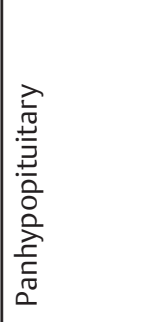 & $\begin{array}{l}0 \\
0 \\
0\end{array}$ & $\begin{array}{l}0 \\
0 \\
0\end{array}$ & 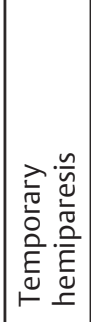 & $\begin{array}{l}0 \\
0 \\
0\end{array}$ & $\begin{array}{l}0 \\
0 \\
0\end{array}$ & 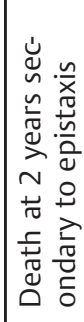 & $\begin{array}{l}8 \\
: \\
0\end{array}$ & 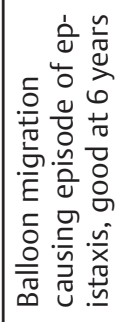 & $\begin{array}{l}0 \\
\\
0\end{array}$ & $\begin{array}{l}0 \\
0 \\
0 \\
0\end{array}$ & 兰 \\
\hline 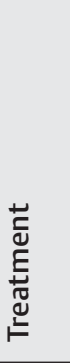 & 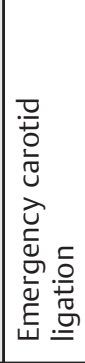 & 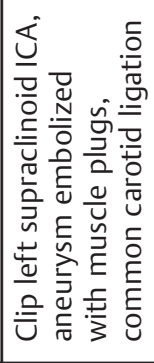 & 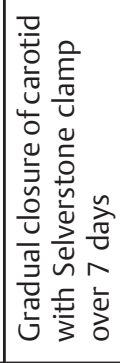 & 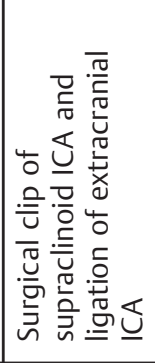 & 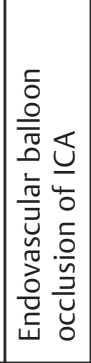 & 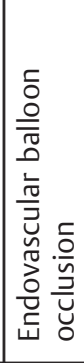 & 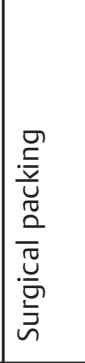 & 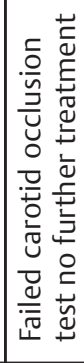 & 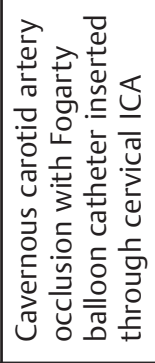 & 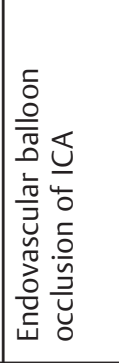 & 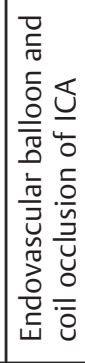 & 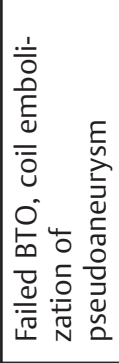 & 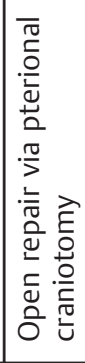 \\
\hline 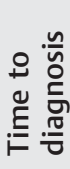 & 部 & $\begin{array}{l}\frac{n}{\pi} \\
0 \\
0\end{array}$ & \begin{tabular}{|l} 
王 \\
号 \\
E \\
-
\end{tabular} & \begin{tabular}{|l}
$\tilde{y}$ \\
$\tilde{y}$ \\
$\tilde{u}$ \\
0
\end{tabular} & $\begin{array}{l}\stackrel{n}{\widehat{\pi}} \\
\frac{\pi}{\sigma} \\
\sigma\end{array}$ & 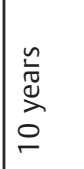 & $\begin{array}{l}\stackrel{n}{\vec{\sigma}} \\
\stackrel{0}{0} \\
0\end{array}$ & 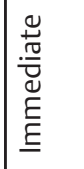 & 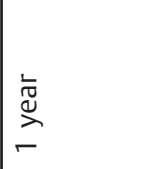 & 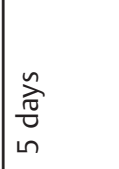 & 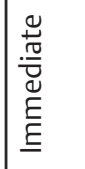 & 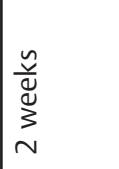 & 告 \\
\hline 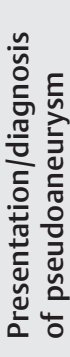 & 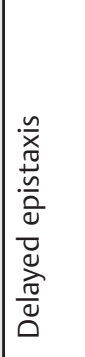 & 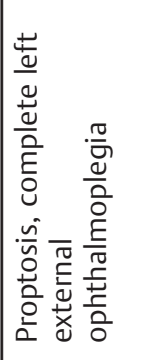 & 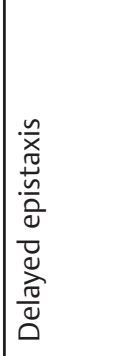 & 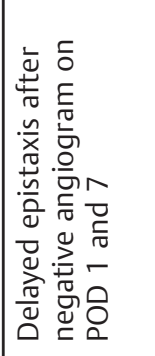 & 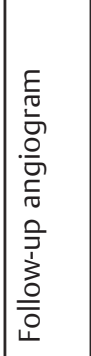 & 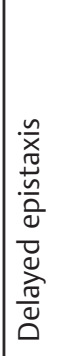 & 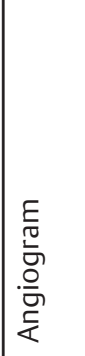 & 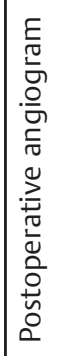 & 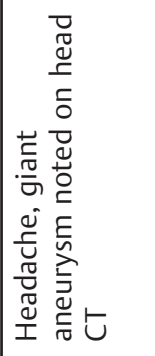 & 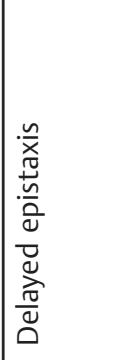 & 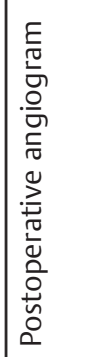 & 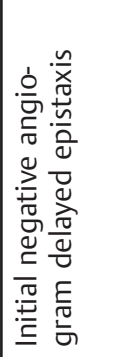 & 妾 \\
\hline 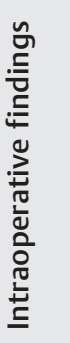 & 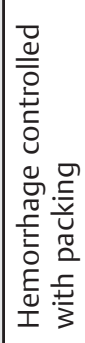 & 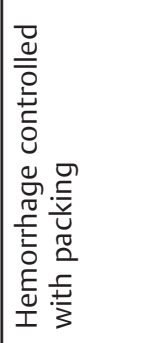 & 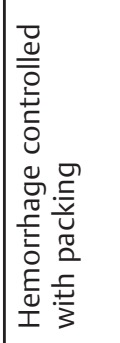 & 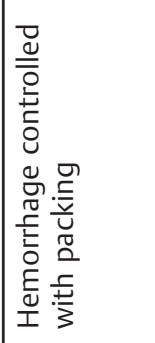 & 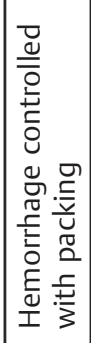 & 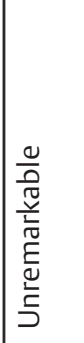 & 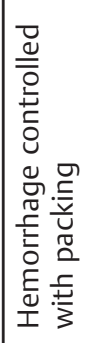 & 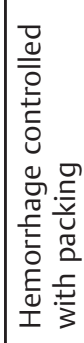 & 兰 & 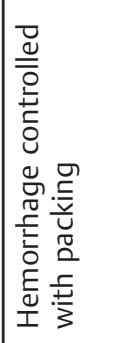 & 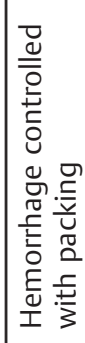 & 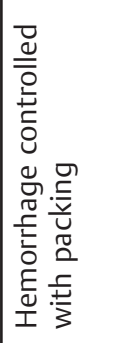 & 兰 \\
\hline$\frac{\circ}{y}$ & 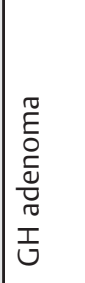 & 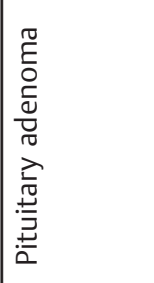 & 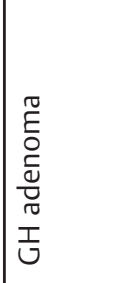 & 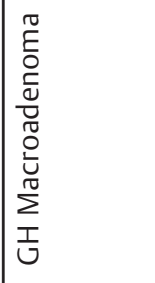 & 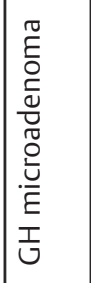 & 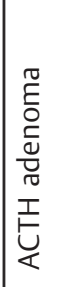 & 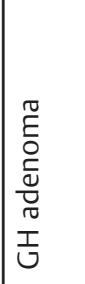 & 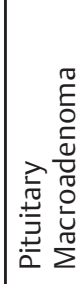 & 产 & 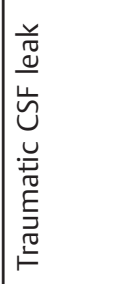 & 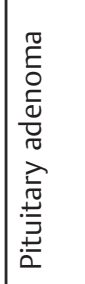 & 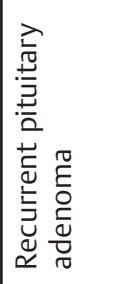 & 兰 \\
\hline$\frac{\frac{\tilde{u}}{\Delta}}{\frac{\sigma}{\alpha}}$ & $\frac{\alpha}{z}$ & $\underset{\substack{\infty \\
+\infty}}{\infty}$ & $\frac{u}{\sigma}$ & 岕 & 岕 & 崖 & $\underset{\infty}{\infty}$ & 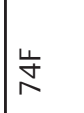 & 岁 & $\stackrel{\stackrel{\leftrightarrow}{\sim}}{\sim}$ & 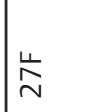 & $\sum_{\tilde{\sigma}}$ & 兰 \\
\hline & 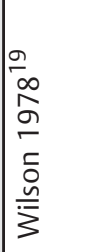 & 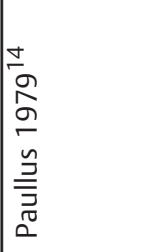 & 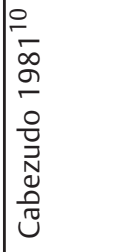 & 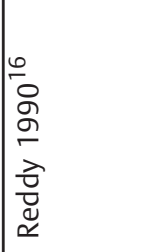 & 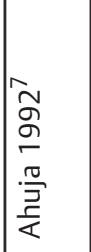 & 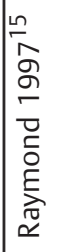 & 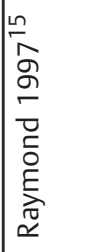 & 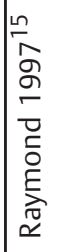 & 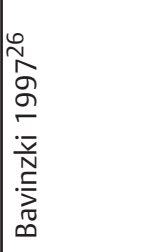 & 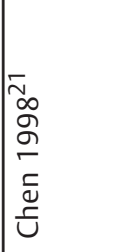 & 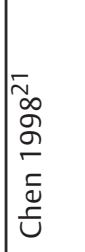 & 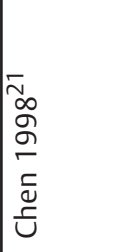 & 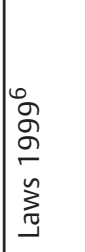 \\
\hline
\end{tabular}




\begin{tabular}{|c|c|c|c|c|c|c|c|c|c|c|c|c|}
\hline 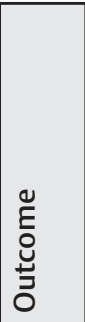 & $\begin{array}{l}0 \\
8 \\
\end{array}$ & 产 & $\begin{array}{l}0 \\
0 \\
0\end{array}$ & $\begin{array}{l}0 \\
8 \\
\end{array}$ & ర & 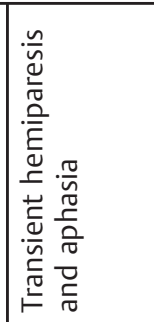 & O & $\begin{array}{l}0 \\
8 \\
0\end{array}$ & $\begin{array}{l}0 \\
\circ \\
\end{array}$ & 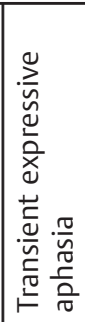 & 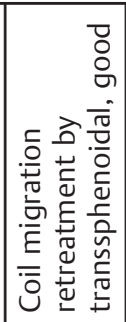 & 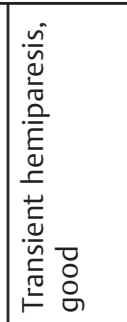 \\
\hline 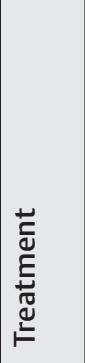 & 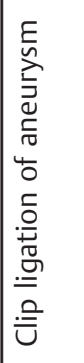 & 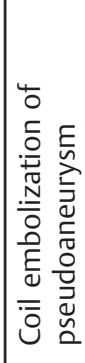 & 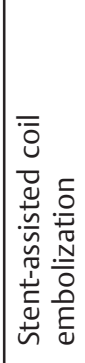 & 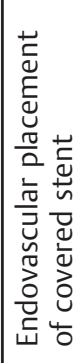 & 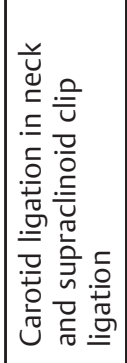 & 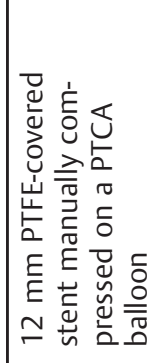 & 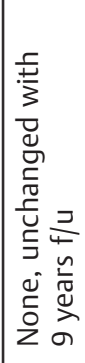 & 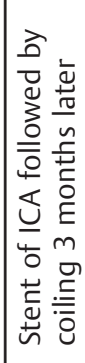 & 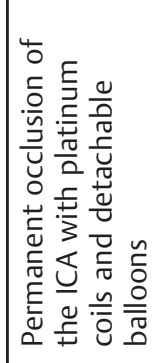 & 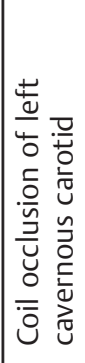 & 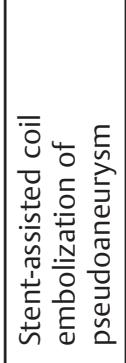 & 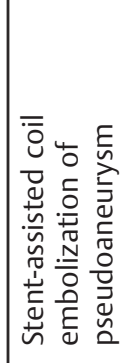 \\
\hline 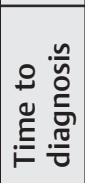 & $\begin{array}{l}\text { 离 } \\
\text { m } \\
m\end{array}$ & 告 & \begin{tabular}{|l}
$\tilde{\ddot{\Xi}}$ \\
$\tilde{\Xi}$ \\
Ln
\end{tabular} & 告 & $\begin{array}{l}\tilde{\widetilde{\sigma}} \\
\stackrel{\tilde{\sigma}}{0} \\
\sim\end{array}$ & $\begin{array}{l}\widetilde{n} \\
\tilde{\sigma} \\
\sim\end{array}$ & $\begin{array}{l}\underset{\hat{\widehat{\sigma}}}{0} \\
0 \\
-\end{array}$ & 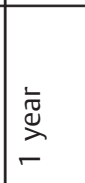 & 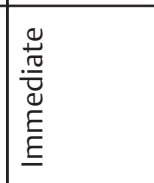 & \begin{tabular}{|l}
$\tilde{y}$ \\
$\tilde{\Xi}$ \\
$\tilde{\nabla}$ \\
$\sigma$
\end{tabular} & $\begin{array}{l}\frac{n}{\sqrt{0}} \\
0 \\
-\end{array}$ & \begin{tabular}{|l}
$\tilde{\check{u}}$ \\
$\tilde{u}$ \\
$\tilde{u}$ \\
\end{tabular} \\
\hline 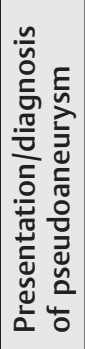 & 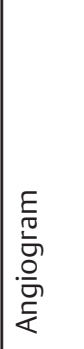 & 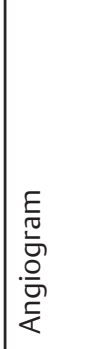 & 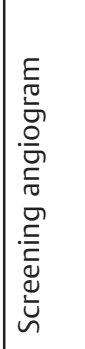 & 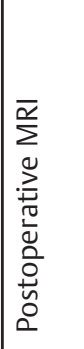 & 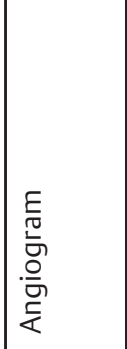 & 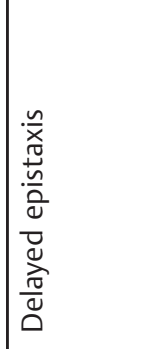 & 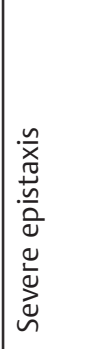 & 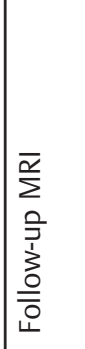 & 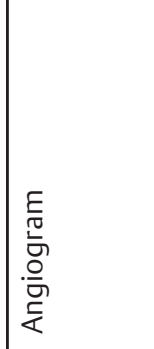 & 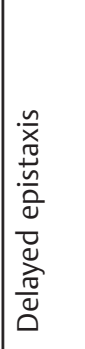 & 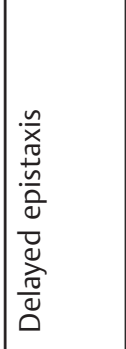 & 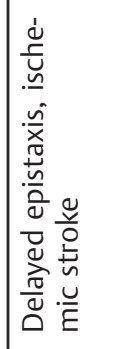 \\
\hline 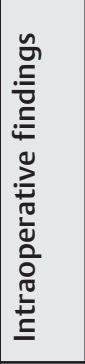 & 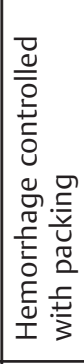 & 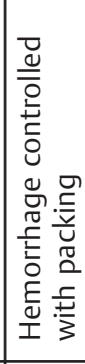 & 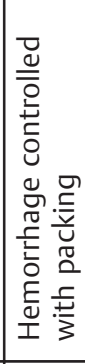 & 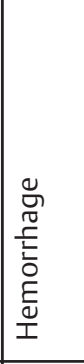 & 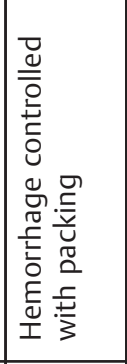 & 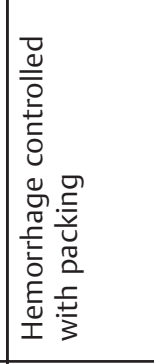 & 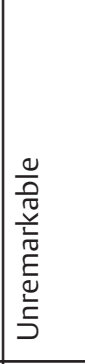 & 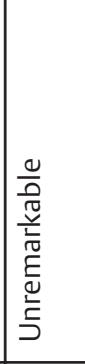 & 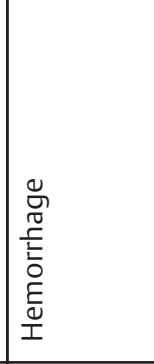 & 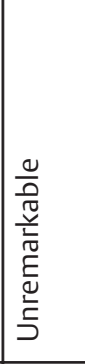 & 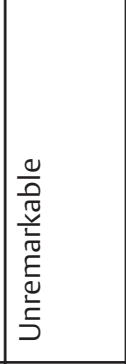 & 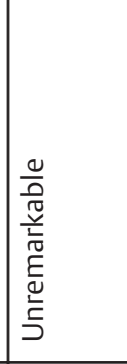 \\
\hline .0 & 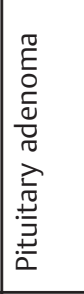 & 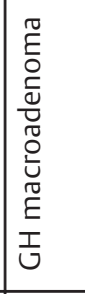 & 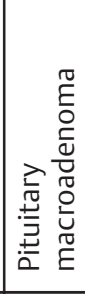 & $\begin{array}{l}0 \\
\tilde{E} \\
0 \\
\tilde{U} \\
\tilde{U} \\
\tilde{T} \\
\tilde{J} \\
\end{array}$ & 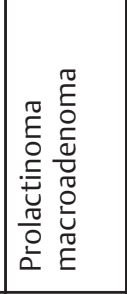 & 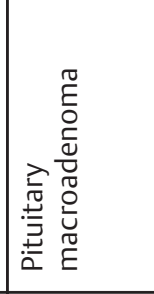 & 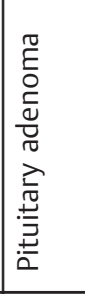 & 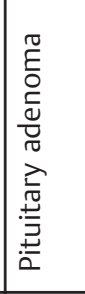 & 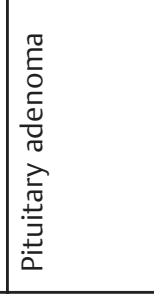 & 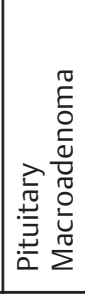 & 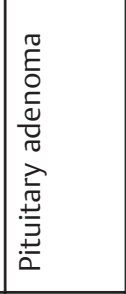 & 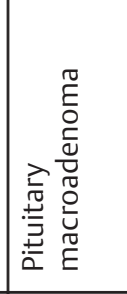 \\
\hline 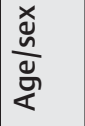 & 嵩 & $\stackrel{\Delta}{N}$ & ¿̇ & $\begin{array}{l}\Delta \\
\infty \\
m\end{array}$ & 仿 & 岕 & $\sum_{\text {L }}$ & $\begin{array}{l}L \\
\text { L } \\
m\end{array}$ & 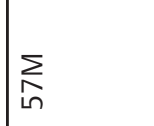 & $\sum_{\text {L }}$ & 方 & 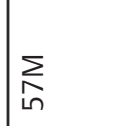 \\
\hline & 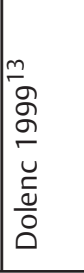 & 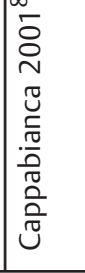 & 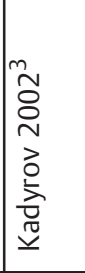 & 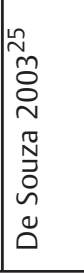 & 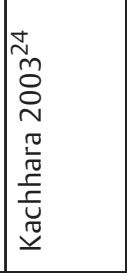 & 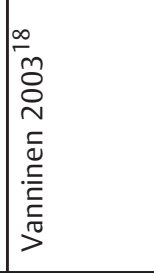 & 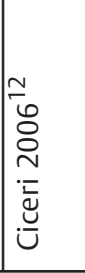 & 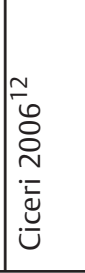 & 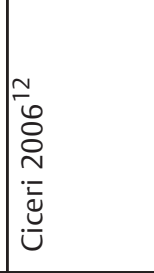 & 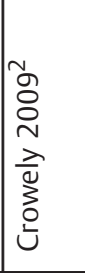 & 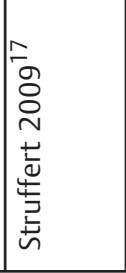 & 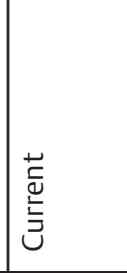 \\
\hline
\end{tabular}


a patient refused surgical management resulted in death at 2 years secondary to uncontrollable epistaxis. ${ }^{15}$ Despite reports that conservatively managed postprocedure femoral pseudoaneurysms spontaneously close in $89 \%$ of patients, we recommend treatment even in incidental false aneurysms of the carotid because of the higher morbidity associated with carotid rupture. ${ }^{22}$

Additional surgical options for cavernous carotid pseudoaneurysms include extracranial to intracranial (EC-IC) bypass with carotid occlusion, craniotomy with direct repair of the carotid artery within the cavernous sinus, or trapping the aneurysm via cervical carotid ligation and supraclinoid carotid clip ligation. ${ }^{6,13,15,23,24}$ Endovascular options may include balloon occlusion of the carotid artery, coil embolization of the pseudoaneurysm or carotid artery, and/or placement of a flow-diverting stent. $3,7,8,12,15,18$ With the advancement in endovascular techniques, open surgery has become less common. Successful carotid artery occlusion, aneurysm trapping, stenting, or coiling can be achieved without subjecting the patient to the risks associated with open craniotomy and manipulation of a cavernous carotid pseudoaneurysm.

Some authors prefer carotid occlusion, when feasible, to avoid the risks associated with manipulation of the false aneurysm. Coil migration of cavernous pseudoaneurysms has been reported. Struffert described two cases requiring repeat transsphenoidal surgery to retrieve coils that had migrated from a pseudoaneurysm into the patients' nostril. ${ }^{17}$ The use of a flow-iverting stent may obviate the need for an intra-aneurysmal embolization. ${ }^{18,25}$ However, flow-diverting stents surrender future endovascular access to the pseudoaneurysm, because once deployed a microcatheter cannot be passed through it. If the patient experiences breakthrough bleeding, he or she could not be treated with further embolic agents.

\section{Conclusion}

Although rare, erosion of an absorbable plate used to reconstruct the bony sellar floor may cause a carotid artery pseudoaneurysm, potentially resulting in a subacute presentation of epistaxis or embolic stroke. The authors' preference is to avoid the use of rigid plating for sellar floor reconstruction in the absence of intraoperative CSF leaks, unless required to buttress a large skull base defect and CSF leak repair construct. Short-segment embolization with stent coiling is the preferred option for the treatment of iatrogenic carotid pseudoaneurysms following endonasal skull base operations.

\section{References}

1 Cappabianca P, Cavallo LM, Colao A, de Divitiis E. Surgical complications associated with the endoscopic endonasal transsphenoidal approach for pituitary adenomas. J Neurosurg 2002;97 (2):293-298

2 Crowley RW, Dumont AS, Jane JA Jr. Bilateral intracavernous carotid artery pseudoaneurysms as a result of sellar reconstruction during the transsphenoidal resection of a pituitary macroadenoma: case report. Minim Invasive Neurosurg 2009;52(1): $44-48$
3 Kadyrov NA, Friedman JA, Nichols DA, Cohen-Gadol AA, Link MJ, Piepgras DG. Endovascular treatment of an internal carotid artery pseudoaneurysm following transsphenoidal surgery. Case report. J Neurosurg 2002;96(3):624-627

4 Berker M, Aghayev K, Saatci I, Palaoğlu S, Onerci M. Overview of vascular complications of pituitary surgery with special emphasis on unexpected abnormality. Pituitary 2010;13(2): 160-167

5 Ciric I, Ragin A, Baumgartner C, Pierce D. Complications of transsphenoidal surgery: results of a national survey, review of the literature, and personal experience. Neurosurgery 1997;40(2): 225-236, discussion 236-237

6 Laws ER Jr. Vascular complications of transsphenoidal surgery. Pituitary 1999;2(2):163-170

7 Ahuja A, Guterman LR, Hopkins LN. Carotid cavernous fistula and false aneurysm of the cavernous carotid artery: complications of transsphenoidal surgery. Neurosurgery 1992;31(4):774-778, discussion 778-779

8 Cappabianca P, Briganti F, Cavallo LM, de Divitiis E. Pseudoaneurysm of the intracavernous carotid artery following endoscopic endonasal transsphenoidal surgery, treated by endovascular approach. Acta Neurochir (Wien) 2001;143(1):95-96

9 Dusick JR, Esposito F, Malkasian D, Kelly DF. Avoidance of carotid artery injuries in transsphenoidal surgery with the Doppler probe and micro-hook blades. Neurosurgery 2007;60(4, Suppl 2):322328, discussion 328-329

10 Cabezudo JM, Carrillo R, Vaquero J, Areitio E, Martinez R. Intracavernous aneurysm of the carotid artery following transsphenoidal surgery. Case report. J Neurosurg 1981;54(1):118-121

11 Camp PE, Paxton HD, Buchan GC, Gahbauer H. Vasospasm after transsphenoidal hypophysectomy. Neurosurgery 1980;7(4):382-386

12 Ciceri EF, Regna-Gladin C, Erbetta A, et al. Iatrogenic intracranial pseudoaneurysms: neuroradiological and therapeutical considerations, including endovascular options. Neurol Sci 2006;27 (5):317-322

13 Dolenc VV, Lipovsek M, Slokan S. Traumatic aneurysm and carotidcavernous fistula following transsphenoidal approach to a pituitary adenoma: treatment by transcranial operation. Br J Neurosurg 1999;13(2):185-188

14 Paullus WS, Norwood CW, Morgan HW. False aneurysm of the cavernous carotid artery and progressive external ophthalmoplegia after transsphenoidal hypophysectomy. Case report. J Neurosurg 1979;51(5):707-709

15 Raymond J, Hardy J, Czepko R, Roy D. Arterial injuries in transsphenoidal surgery for pituitary adenoma; the role of angiography and endovascular treatment. AJNR Am J Neuroradiol 1997;18 (4):655-665

16 Reddy K, Lesiuk H, West M, Fewer D. False aneurysm of the cavernous carotid artery: a complication of transsphenoidal surgery. Surg Neurol 1990;33(2):142-145

17 Struffert T, Buhk JH, Buchfelder M, Rohde V, Doerfler A, Knauth M. Coil migration after endovascular coil occlusion of internal carotid artery pseudoaneurysms within the sphenoid sinus. Minim Invasive Neurosurg 2009;52(2):89-92

18 Vanninen RL, Manninen HI, Rinne J. Intrasellar latrogenic carotid pseudoaneurysm: endovascular treatment with a polytetrafluoroethylene-covered stent. Cardiovasc Intervent Radiol 2003; 26(3):298-301

19 Wilson CB, Dempsey LC. Transsphenoidal microsurgical removal of 250 pituitary adenomas. J Neurosurg 1978;48(1):13-22

20 Cappabianca P, Cavallo LM, Esposito F, Valente V, De Divitiis E. Sellar repair in endoscopic endonasal transsphenoidal surgery: results of 170 cases. Neurosurgery 2002;51(6):1365-1371, discussion 1371-1372

21 Chen D, Concus AP, Halbach VV, Cheung SW. Epistaxis originating from traumatic pseudoaneurysm of the internal carotid artery: diagnosis and endovascular therapy. Laryngoscope 1998;108(3): 326-331 
16 Cavernous Carotid Artery Pseudoaneurysm Following Surgery Tuchman et al.

22 Toursarkissian B, Allen BT, Petrinec D, et al. Spontaneous closure of selected iatrogenic pseudoaneurysms and arteriovenous fistulae. J Vasc Surg 1997;25(5):803-808, discussion 808-809

23 Charbel FT, Gonzales-Portillo G, Hoffman W, Cochran E. Distal internal carotid artery pseudoaneurysms: technique and pitfalls of surgical management: two technical case reports. Neurosurgery 1999;45(3):643-648, discussion 648-649

24 Kachhara R, Menon G, Bhattacharya RN, et al. False aneurysm of cavernous carotid artery and carotid cavernous fistula: complica- tions following transsphenoidal surgery. Neurol India 2003;51(1): 81-83

25 de Souza JM, Domingues FS, Espinosa G, Gadelha M. Cavernous carotid artery pseudo-aneurysm treated by stenting in acromegalic patient. Arq Neuropsiquiatr 2003;61(2B): 459-462

26 Bavinzski G, Killer M, Knosp E, Ferraz-Leite H, Gruber A, Richling B. False aneurysms of the intracavernous carotid artery-report of 7 cases. Acta Neurochir (Wien) 1997;139(1):37-43 